livraisons

d'Histoire

de l'Architecture

\section{Livraisons de l'histoire de l'architecture}

13 | 2007

Architectures des établissements d'enseignement supérieur

\title{
La doctrine de la restauration en France entre les deux guerres : quelques considérations sur la mise à jour de la discipline
}

The doctrine of restoration in France between the two World Wars: some

thoughts on the revised tenets

Die Theorie der Restaurierung in Frankreich während der Zwischenkriegszeit :

einige Betrachtungen über die Aktualisierung der Disziplin

\section{Stéphane Garnero}

\section{OpenEdition}

Journals

Édition électronique

URL : http://journals.openedition.org/lha/416

DOI : 10.4000//ha.416

ISSN : 1960-5994

Éditeur

Association Livraisons d'histoire de l'architecture - LHA

Édition imprimée

Date de publication : 10 juin 2007

Pagination : $123-136$

ISSN : 1627-4970

Référence électronique

Stéphane Garnero, «La doctrine de la restauration en France entre les deux guerres : quelques

considérations sur la mise à jour de la discipline », Livraisons de l'histoire de l'architecture [En ligne],

13 | 2007, mis en ligne le 10 juin 2009, consulté le 19 avril 2019. URL : http://journals.openedition.org/ Iha/416; DOI : 10.4000/lha.416

Ce document a été généré automatiquement le 19 avril 2019

Tous droits réservés à l'Association LHA 


\title{
La doctrine de la restauration en France entre les deux guerres : quelques considérations sur la mise à jour de la discipline ${ }^{1}$
}

\author{
The doctrine of restoration in France between the two World Wars: some \\ thoughts on the revised tenets \\ Die Theorie der Restaurierung in Frankreich während der Zwischenkriegszeit : \\ einige Betrachtungen über die Aktualisierung der Disziplin
}

Stéphane Garnero

1 Les années comprises entre les deux guerres mondiales constituent une période fondamentale pour le développement de la culture internationale de la restauration: d'une part, les techniques du XIX sont définitivement dépassées, d'autre part, de nouvelles perspectives s'ouvrent, préfigurant souvent des thèmes aujourd'hui encore largement débattus².

2 La France, dont les architectes ont joué un rôle essentiel dans le développement de la théorie de la restauration tout au long du XIX ${ }^{e}$ siècle, voit dans ces années se confirmer son rôle de leader international, même si beaucoup d'autres acteurs se présentent sur scène, avec des positions souvent divergentes. La recherche de bases solides et admises par tous, sur lesquelles pourrait se développer la discipline, conduit en définitive à un rapprochement des différentes positions nationales, qui, tout en maintenant des spécificités, apparaissent moins dissemblables après analyse.

3 La lecture des procès-verbaux des réunions de la commission des monuments historiques permet de découvrir les moments privilégiés qui amènent la doctrine française à s'intéresser à des problématiques souvent considérées comme étant l'apanage d'autres nations, culturellement plus proches des thèses de la conservation. La « restauration » et les «monuments » ne concentrent plus, comme au siècle précédent, les soucis de l'action de tutelle, mais laissent la place à de nouveaux centres d'intérêt, comme la 
"conservation", avec toutes les nuances qu'une telle catégorie peut comporter, et l'« environnement », qui devient lui-même objet de conservation ${ }^{3}$.

\section{Le débat à la Conférence internationale d'Athènes}

Dans son rapport à la Conférence d'Athènes, en 1931, Gustavo Giovannoni tente d'affirmer les positions italiennes à l'échelon international, en théorisant les principes qui avaient déjà été exposés par Camillo Boito en 1883, au $\mathrm{II}^{\mathrm{e}}$ congrès des ingénieurs et architectes italiens ${ }^{4}$. En particulier, il insiste sur deux aspects fondamentaux : le postulat de la distinction des matériaux à travers l'«utilisation, pour combler les lacunes et redresser les lignes, de matériaux nouveaux » afin de « distinguer les éléments ajoutés des éléments originels, de façon à ne pas créer de faux artistiques et à ne pas induire les savants en erreur", et le principe de la simplicité des adjonctions, qui doivent être réalisées "sans aucune prétention ornementale » uniquement «à travers des effets de masse $»^{5}$. Son analyse se poursuit par la critique des possibilités expressives de l'architecture contemporaine, celle-ci étant considérée comme un phénomène de «mode» et donc «absolument incompatible avec les expressions permanentes de l'architecture, [...] encore plus quand on se trouve en présence de restes vénérables $d u$ passé $»^{6}$. Ainsi, les interventions modernes ne peuvent pas être apparentes et doivent se concentrer sur « les éléments qui n'entrent pas dans l'aspect général de l'édifice antique » ${ }^{7}$ : l'apport de l'architecture contemporaine est ainsi limité de façon drastique, cantonné aux parties invisibles des constructions. Toutefois, les principes de la différenciation des matériaux, de la simplification des adjonctions et de l'abandon de l'architecture contemporaine ne sont pas repris dans les conclusions de la Conférence d'Athènes, conclusions qui ne font aucune mention des dispositions giovannoniennes, mais se limitent à condamner « les restitutions intégrales » et à promouvoir l'« entretien régulier et permanent propre à assurer la conservation des édifices $»^{8}$. Le principe de la distinction des adjonctions est repris exclusivement pour les opérations d'anastylose des monuments archéologiques, en considérant que «les matériaux nouveaux nécessaires à cet effet devraient être toujours reconnaissables $»^{9}$.

\section{Le concept d'invisibilité}

5 En France, les principes de la restauration, exposés par Charles Genuys dans l'immédiat après-guerre, alors que les débats étaient encore centrés sur la méthode plutôt que sur l'activité concrète, tentent de s'aligner sur certains points des théories giovannoniennes. En s'interrogeant sur les modalités de reconstruction des églises endommagées par les bombardements, Genuys «exprime l'avis que les réparations nécessitées par leur état actuel ne devront pas être trop complètes et qu'il conviendra de limiter ces réparations de façon à conserver aux édifices les traces des bombardements subis par eux, tout en assurant bien entendu leur conservation $»^{10}$. Néanmoins, ces théories n'ont un résultat positif que lorsque peu de parties sont endommagées : si les altérations sont importantes, c'est l'esthétique du monument qui prime pour éviter l'illisibilité de l'architecture dans son ensemble.

Une fois les chantiers démarrés, on se rend vite compte que les principes de la différenciation des matériaux et de la simplicité des éléments additionnels ne sont pas 
applicables aux monuments gravement endommagés par la guerre, qui doivent revivre au plus vite : les positions théoriques sont abandonnées pour assurer d'abord la réparation des destructions subies. Les archéologues eux-mêmes qui s'opposent, dans un premier temps, au principe de la « restauration invisible $»^{11}$, particulièrement en ce qui concerne les parties ornées des édifices, doivent se plier aux nécessités de la reconnaissance collective. C'est ce qui s'est produit à Reims où les demandes de Lefèvre-Pontalis qui visent en un premier temps à la conservation de tous les dommages de guerre, y compris les traces des bombes sur les murs, sont suivies de reconstructions de parties décorées. Le président de la Société française d'archéologie, favorable depuis toujours à la conservation la plus sévère, tente d'établir alors une règle pour justifier la reconstruction en cours : contre l'avis d'Auguste Rodin et d'Edmond Rostand qui veulent laisser, comme à Jumièges, l'édifice en ruine, il propose une reconstruction au nom de la vie spirituelle et matérielle des vivants ${ }^{12}$ (ill. 1).

III. : Cathédrale de Reims, détail des décorations de la façade après les bombardements

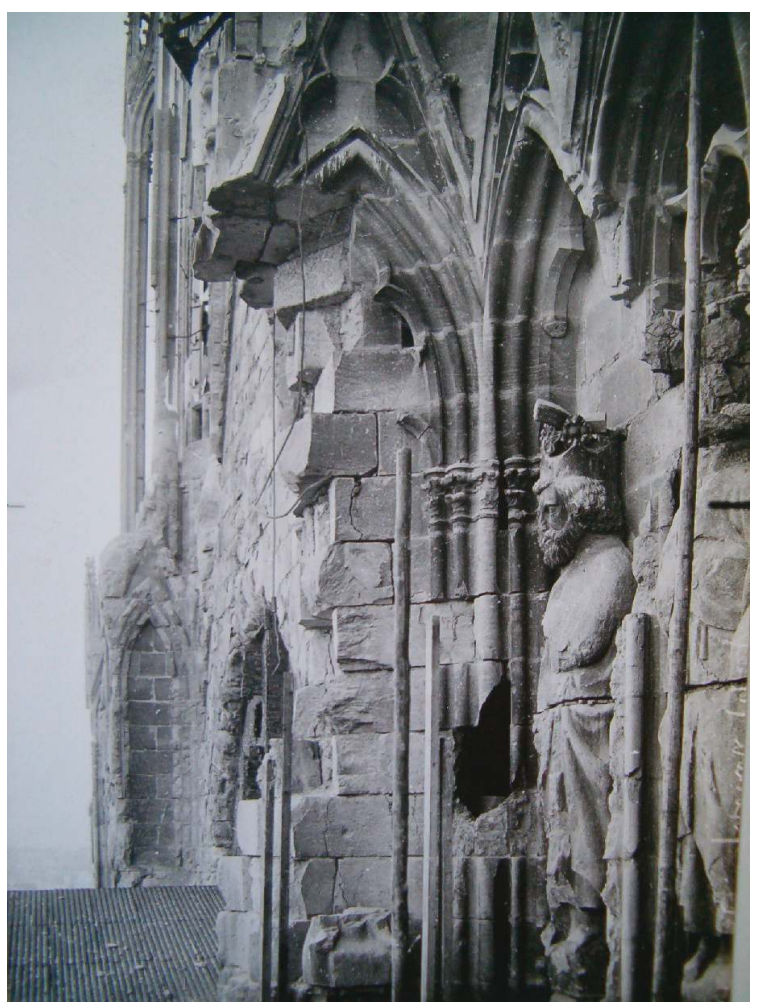

AMP Fo U 144

Cathédrale de Reims, album de photographies prises au cours des travaux de restauration, 1921.

7 Si l'intervention de reconstruction, réalisée à la suite d'un événement traumatisant, peut être justifiée par des nécessités sociales et culturelles supérieures ${ }^{13}$, il n'en est pas autant d'une certaine pratique généralisée visant à l'« invisibilité » des œuvres. Il est à noter que la politique de sauvegarde du service des monuments historiques, à partir des premières années $\mathrm{du} \mathrm{XX}^{\mathrm{e}}$ siècle, est en premier lieu centrée sur l'entretien continu des édifices moyennant la création du corps des " architectes ordinaires ", domiciliés sur le territoire, et l'affectation de financements annuels à cet effet ${ }^{14}$.

Dans le cas français, les techniques historiques de la construction interviennent en continuité avec ce qui existe déjà, en conservant les éléments non dégradés et en 
remplaçant les éléments endommagés à l'aide de matériaux, de techniques et de finitions semblables à l'originel. La grande connaissance des architectes et des ouvriers employés dans les travaux d'entretien facilite la réfection de nombreux détails, qui sont plus « compatibles » avec l'architecture existante que les éléments modernes, mais ne sont pas autant visibles et réversibles. La gestion soignée du patrimoine culturel devrait mener, en dernière analyse, à conserver la matière authentique de l'œuvre, et il est nécessaire d'opérer prudemment et avec mesure car, sur une longue période, les entretiens peuvent transformer lentement mais profondément l'œuvre elle-même.

C'est encore plus le cas lorsque l'on restaure des édifices nécessitant des travaux importants. Ces interventions, qui ont un caractère philologique, sont liées à une lecture attentive des traces historiques, mais souvent elles ne se contentent pas de conserver ou de mettre en valeur les empreintes du passé, elles préfèrent les reconstruire. La distinction entre les interventions et l'état de fait est, dans de nombreux cas, entièrement effacée, le résultat recherché étant celui de la continuité historique entre l'œuvre du passé et l'œuvre du présent. Dans le cas des «monuments nationaux », la reconstruction peut aller jusqu'à la recomposition stylistique de nombreuses parties des édifices.

\section{Conservation - innovation}

Cependant dans ses interventions, la commission des monuments historiques s'oppose souvent aux réfections en style et, d'une manière plus générale, aux pastiches, et elle tente d'affirmer une nouvelle théorie qui étend la définition d' " authenticité » au-delà des limites de la distinction giovannonienne entre les apports anciens et modernes. On dénonce ainsi le danger d'une division drastique et d'une opposition entre l'architecture contemporaine et l'architecture historique, en empêchant à la première de résoudre le problème, souvent inéluctable, de la jouissance artistique du bien et en ne laissant à la restauration que les deux orientations suivantes: «celle d'occulter les ouvrages de transformation ou d'adaptation technique et celle, opposée, de les exhiber comme un signe déclaré de différence $»^{15}$.

11 Paul Léon, s'adressant aux jeunes étudiants de l'École de Chaillot qui venait d'être réouverte, exprime ainsi la nécessité de rapprocher l'art moderne de l'art historique, en les fondant pour rechercher de nouveaux terrains d'expression : « Est-il besoin d'ajouter que nous ne prétendons point enfermer les Architectes dans l'étude exclusive du passé ? L'effort des vieux maîtres gothiques pour vaincre la matière inerte, diminuer les points d'appui, couvrir les larges portées, ouvrir de vastes espaces aux colorations de la lumière, n'est-il point celui-là même qui, avec les méthodes et les matériaux de notre industrie moderne, s'impose aux constructeurs d'aujourd'hui ? [...] Chaque siècle est, suivant le vieil adage, un tombeau et un berceau $»^{16}$.

D'après ce point de vue, le concepteur moderne et le restaurateur ${ }^{17}$ doivent se résumer en une seule figure: ce n'est pas par hasard que le premier concours public ouvert aux jeunes professionnels désireux d'assumer la charge d'architecte en chef propose, parmi les autres épreuves d'admission, la réalisation d'un édifice neuf se situant entre deux édifices existants et que le programme demande explicitement que «le pastiche soit écarté et qu'il y ait lieu de rechercher une solution moderne ${ }^{18}$.

13 La position théorique française illustre, par des exemples, la difficulté que les principes de la différenciation des matériaux et de la simplicité des éléments additionnels 
comportent, des résultats apparemment antithétiques étant compris sous le même attribut. Sont « distincts » les marbres moulurés mis en œuvre par Formigé au Trophée de la Turbie, ces derniers ayant été façonnés avec des outils différents des originaux, tout comme l'est l'adjonction moderne conçue par Duval et Gonze à l'église de Saint-Pierre-deRoye.

Dans le premier cas, en réalisant de nouvelles moulures pour le Trophée de la Turbie, l'architecte en chef Jules Formigé prend soin que celles-ci soient sculptées de sorte que «l'on puisse toujours distinguer un bloc ancien d'un bloc moderne en le regardant de près $»^{19}$ (ill. 2).

\section{2 : La Turbie (Alpes-Maritimes)}

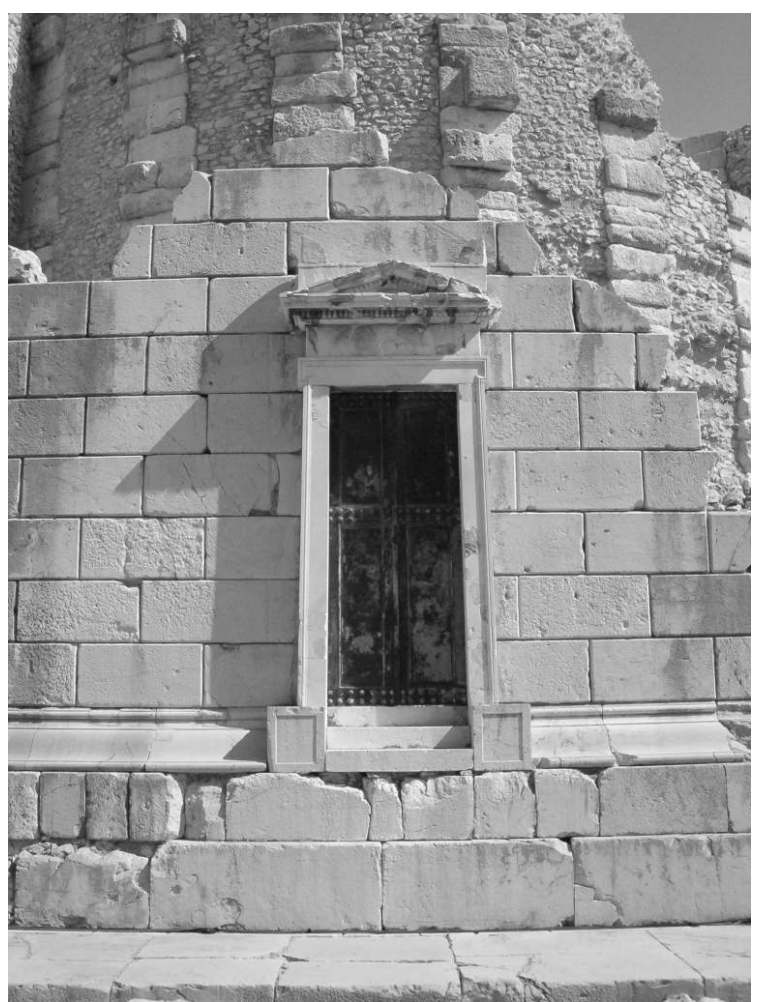

État actuel, détail de l'anastylose des éléments avec des insertions nouvelles

Cl. Stéphane Garnero.

Dans le second cas, en encourageant la reconstruction d'une grande partie de l'église bombardée, la Commission note avec satisfaction le fait que le projet pour la nouvelle structure à adosser au chœur antique, encore existant, adopte un langage contemporain, avec des «formes en fonction de l'art moderne et des procédés nouveaux de construction $»^{20}$, à condition que « toutes dispositions soient prises pour harmoniser cette partie moderne de l'édifice avec le chœur actuellement en cours de restauration $»^{21}$ (ill. 3 et 4). 
III. 3 : Église de Saint-Pierre à Roye

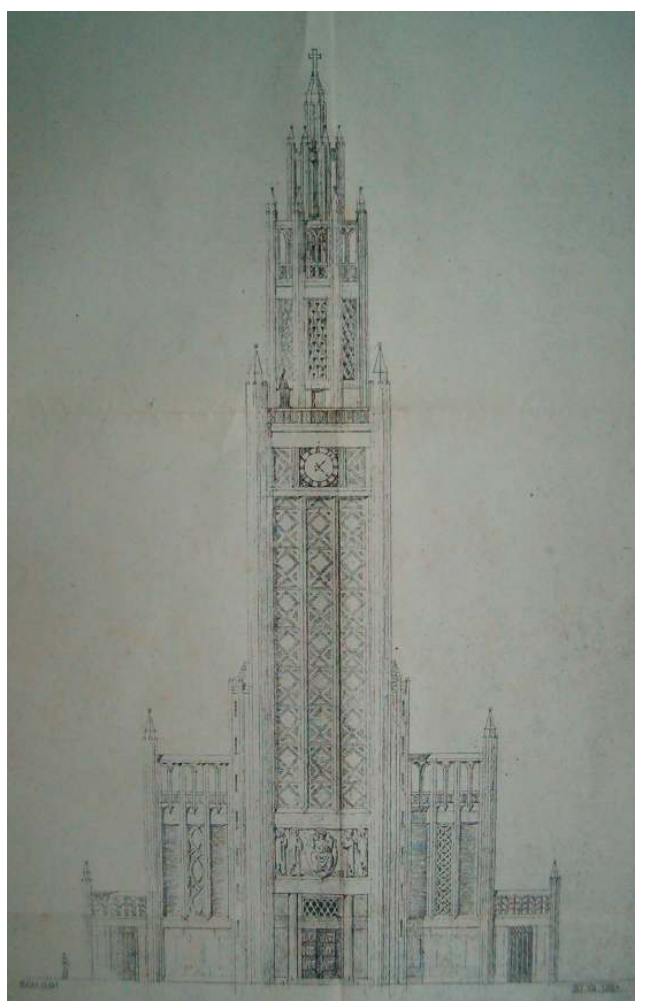

Projet de Duval et Gonze, façade principale AMP 97/40/0862.

\section{4 : Église de Saint-Pierre à Roye}

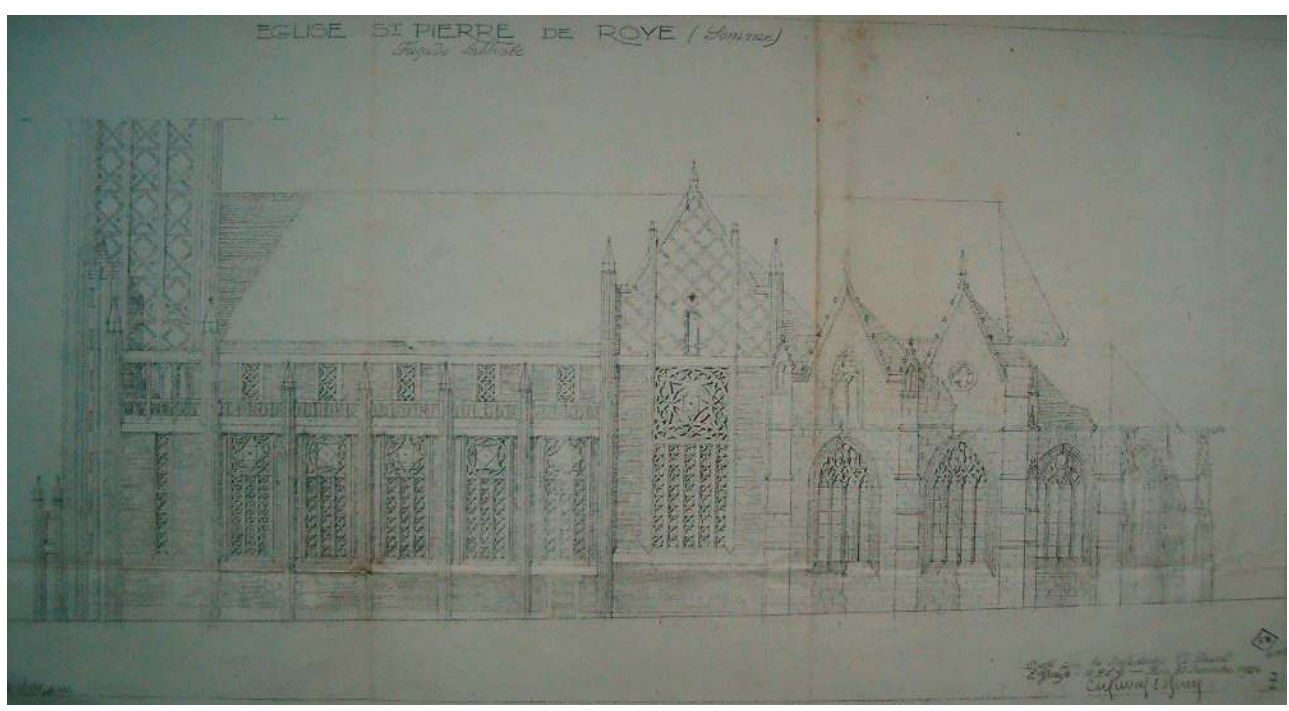

Projet de Duval et Gonze, façade latérale avec la représentation du chœur restauré et du projet AMP 97/40/0862.

16 Ces interventions se placent aux deux extrêmes de la gamme infinie de nuances possibles, dans laquelle la disjonction et l'adaptation, le projet et la conservation se confrontent dans la définition d'un résultat unitaire. 


\section{Différenciation des matériaux - simplicité des éléments - compatibilité - authenticité}

17 L'action de la Commission consiste à faire entrer toutes les interventions dans une "théorie du milieu», en écartant les extrêmes de la recomposition stylistique et de l'opposition moderne anti-historique. Il ne s'agit donc pas d'une créativité liée à la réfection du passé, pas plus que d'une créativité disjointe du contexte: le professionnel doit plutôt se sentir libre de s'adapter à l'antique, en se laissant conduire par l'histoire. Les traits de crayon, minces et légers, des architectes restaurateurs s'opposent ainsi aux pesants signes des architectes du XIX siècle et aux coups de gomme drastiques des architectes modernes. Le travail des architectes du Service des monuments historiques est interprété en clef de projet, leur intervention ne se limite pas à la triade conservationrestauration-réfection, mais va jusqu'à la conception du moderne dans les édifices antiques, à réaliser avec un langage simple, se basant sur la sincérité des matériaux utilisés et l'adaptation des formes aux nécessités de l'emploi ${ }^{22}$.

Cette formule va bien au-delà des dispositions de la théorie giovannonienne qui s'arrête à la recherche de la différenciation des matériaux et de la simplicité des éléments additionnels, fins en soi. Des formes et des formules pré-constituées n'existent pas dans la doctrine française, mais plutôt des orientations ouvertes à l'élaboration d'un nouveau langage architectonique fondé sur l'histoire. Il semble pouvoir dire que la compatibilité du langage ne doive plus, selon ces présupposés, être uniquement dictée par une banale simplicité esthétique, mais puisse aspirer à une compassion plus profonde et plus intime qui est, en premier lieu, compréhension.

19 Les propositions des architectes en chef qui contiennent une forte recomposition stylistique sont généralement refusées; on leur demande d'orienter leurs recherches vers des solutions plus modernes, plus linéaires et plus simples. L'on cite, à titre d'exemple, le cas de l'église de Nasbinals, que l'on a décidé d'agrandir dans le but d'y aménager la nouvelle sacristie, en imposant toutefois «que les adjonctions soient faites très simplement et sans recherche de style, de manière à ne pas se confondre avec les parties anciennes classées ${ }^{23}$, et celui du palais parisien de l'hôtel de Sens, dans lequel le projet est critiqué comme étant un pastiche et pour lequel on suggère d'avoir recours à « une architecture franchement moderne, mais simple, à l'échelle du monument et en harmonie dans le choix des matériaux $»^{24}$. On retrouve ces orientations à propos des travaux concernant le Palais royal à Paris où, en 1933, la galerie de la cour est reconstruite selon un nouveau projet qui reçoit l'assentiment du conseil : «M. Paul Léon n'est nullement hostile à la création d'un décor moderne dans le cadre du Palais Royal qui a, d'ailleurs, été réalisé en plusieurs étapes ${ }^{25}$ (ill. 5). 


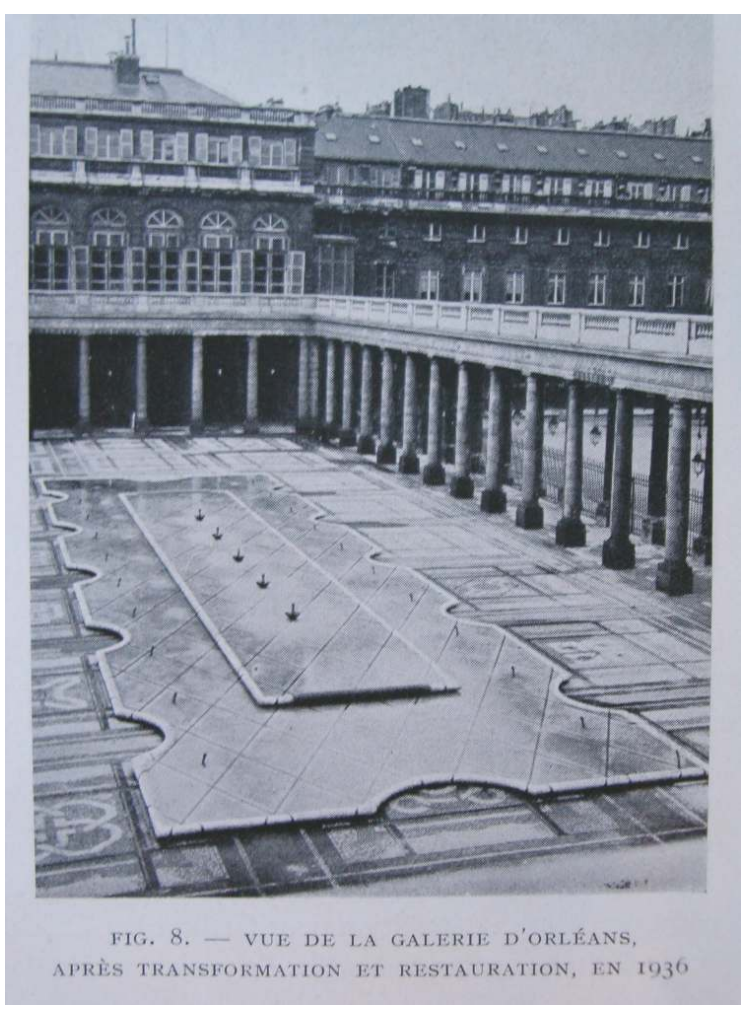

André Ventre, «Les transformations successives du Palais-Royal de 1628 à 1936 »

Dans Les Monuments Historiques de la France, 1936, p. 112-117

À l'opposé, le caractère de la simplicité des adjonctions, bien qu'étant régressif car limitant les capacités de conception des architectes, s'impose pour éviter des dommages plus graves. Les élans de conception de professionnels trop zélés sont ainsi ramenés à une plus grande "compatibilité ». Par exemple, la construction d'un hôtel dans la Cité de Carcassonne est acceptée à condition d'effacer le caractère moderne de sa façade : l'on propose plutôt d'adopter un langage local pour la couleur des façades et les matériaux des couvertures et de supprimer un étage et les balcons de l'édifice ${ }^{26}$.

De plus, la recherche de proportions appropriées au contexte urbain préoccupe beaucoup la Commission : à Paris, le projet pour l'agrandissement de l'ancien hôtel Vendôme devra être proportionné à l'édifice historique en ce qui concerne les dimensions de ses corniches et de ses fenêtres, au bénéfice de sa meilleure insertion ${ }^{27}$. Cette recherche est clairement lisible à propos de la reconstruction des édifices autour de la place centrale de Noyon, entièrement détruits par les bombardements. Paul Léon fait observer qu'il ne faudra pas se laisser aller à une "pure fantaisie d'artiste", mais plutôt « rapprocher le point de vue de l'archéologie et de l'histoire de celui de l'art et de la conception ${ }^{28}$. Mais la leçon du directeur des beaux-arts est difficile à mettre en œuvre et l'on aura recours, dans ce cas, à des «façades absolument neutres pour les nouveaux bâtiments »"

\section{Les sculptures, les vitraux, les arts religieux}

Le thème de la restauration des sculptures qui ornent les cathédrales françaises réintroduit, en les accentuant, les problématiques qui se rapportent à l'architecture. 
André Hallays, à Reims où les statues sont reconstruites, note comme «il importe toutefois que cette restauration ne constitue pas un précédent; la réfection de la statuaire doit être en principe d'autant plus interdite $\aleph^{30}$. Il faut établir une limite au-delà de laquelle la reconstruction ne peut être entreprise, pour ne pas aboutir, malgré les meilleures intentions, à une réfection complète. D'importants exemples montrent l'orientation vers la simplification des formes, comme à Tours où l'on propose d'ébaucher l'ornement de quatre statues au lieu de les reconstruire, " afin de laisser aux statues l'aspect de silhouettes " ${ }^{31}$. Il en est de même dans l'église de Saint-Hilaire à Poitiers où les chapiteaux, n'existant plus, ne sont qu'ébauchés pour créer une forme géométrique qui ne recherche aucune composition décorative. À Versailles, la restauration des fresques du Salon du Roi et du Salon carré doit permettre « le maintien de la peinture existante. Une trace à la sanguine restituera les contours de la décoration disparue, suivant le procédé en usage en Italie et en Allemagne $»^{32}$. Ces exemples sont néanmoins souvent critiqués, du fait de leur profond manque de valeurs artistiques ${ }^{33}$. Dans d'autres cas, on s'oriente vers une composition moderne : les sculptures de l'église de Saint-Gervais à Paris, œuvres du milieu du XIX ${ }^{e}$ siècle, sont remplacées non par des copies, mais par des figures de conception moderne, inspirées simplement des représentations historiques. Il ne s'agit pas d'opérer un pastiche, en recréant le décor de la façade de 1616, mais plutôt d'intervenir avec une sensibilité plus grande que celle du XIX ${ }^{e}$ siècle et de créer une

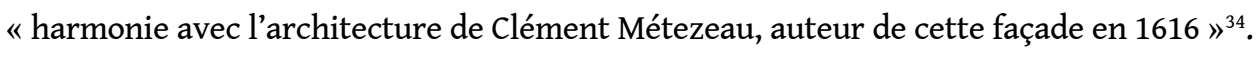

Le cas des vitraux et des arts religieux est encore plus clair : la reconstruction présente de nombreux exemples où les architectes proposent des solutions modernes, bien que très bien intégrées dans l'architecture qui les accueille. Le développement d'une nouvelle sensibilité semble apparaître, à tel point que Paul Deschamps se demande " pourquoi ne pas faire appel aux verriers modernes [...] ? L'essentiel est que ces œuvres nouvelles s'adaptent à l'harmonie générale du monument où elles doivent prendre place $»^{35}$. Ainsi le nouvel autel pour le Mont-Saint-Michel, dessiné en 1926 par Pierre Paquet, «a été étudié pour qu'il ne puisse nuire, ni par sa forme, ni par son importance, au caractère de l'église. [...] Le bronze sera doré et patiné, le granit proviendra des carrières de Bourgogne, il a été spécialement choisi pour s'harmoniser avec le ton général des murs » ${ }^{36}$ (ill. 6). 


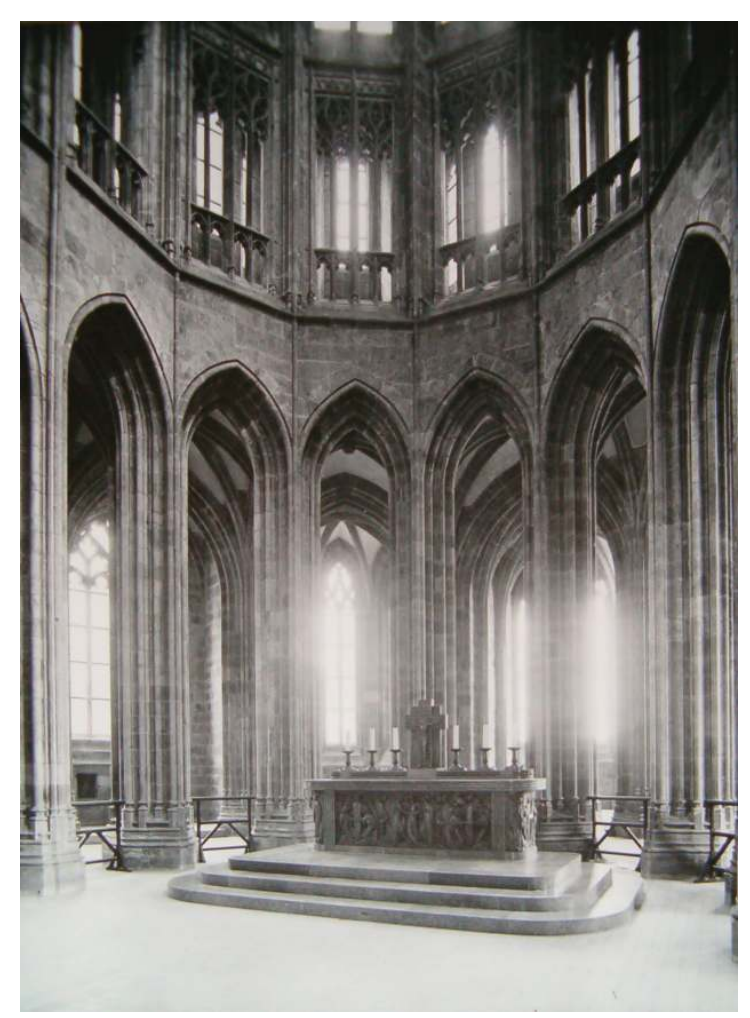

Autel, projet de Pierre Parquet

AMP 119669

\section{Conservation, mais non restauration}

$\mathrm{Au}$ sein de la commission des monuments historiques se dessine donc une forte et solide doctrine qui s'interroge sur les grandes questions en débat, encourageant des solutions souvent innovantes. Paul Léon, directeur général des beaux-arts et à la tête de la Commission pendant de nombreuses années, synthétise l'ensemble des transformations en parlant d'une phase " expérimentale, celle de la conservation », et ajoute que « depuis la fin du dernier siècle les architectes, renonçant à reconstruire les édifices tels qu'ils ont dû exister, se bornent à les maintenir tels que nous les a légués le passé. La restauration fait place à la conservation ${ }^{37}$.

Le thème de la conservation, entendue comme l'ensemble des opérations nécessaires pour assurer une transmission du patrimoine aux futures générations, devient la clef de lecture commune à toutes les interventions de restauration, effectuée à travers un entretien attentif de l'œuvre et le respect de toutes les phases historiques de l'édifice. Comme le dit Charles Genuys, inspecteur général des monuments, "l'architecte des monuments historiques doit être beaucoup plus médecin que chirurgien. La médecine, c'est le strict, le sévère entretien. La chirurgie, il ne faut y recourir que dans les cas graves, pressants, urgents quand l'existence d'un édifice est en péril, en cas par exemple, de désagrégation de ses parties maîtresses, et c'est cette manière de voir que je voudrais inculquer à nos futurs architectes. Conservation, mais non restauration ${ }^{38}$. 


\section{NOTES}

1. La présente étude dérive de la thèse de doctorat en restauration: Conservazione e restauro in Francia. 1919-1939: i lavori della "Commission des monuments historiques", Faculté d'architecture, École polytechnique de Turin (Italie), 2004. Mes remerciements vont à Maurizio Momo et François Loyer (directeurs de thèse), Françoise Choay, Tatiana Kirova, Benjamin Mouton, Alexandre Melissinos, Jida Vincent et Jean-Daniel Pariset.

2. Françoise Choay, La Conférence d'Athènes sur la conservation artistique et historique des monuments (1931), Paris, Les Éditions de l'Imprimeur, 2002.

3. Le thème de l'environnement, du territoire et de la ville, qui n'est pas développé ici, représente lui aussi une nouveauté substantielle au sein des positions théoriques internationales.

4. Giovannoni reconnaît en Boito « le plus influent et persévérant défenseur » des principes de la restauration italienne. Camillo Boito, Ordine del giorno sul restauro, Convegno Nazionale degli Ingegneri e Architetti Italiani, Roma 1883 ; voir aussi Camillo Boito, Conserver ou restaurer: les dilemmes du patrimoine, les Éd. de l'Imprimeur, Besançon 2000, préface de Françoise Choay.

5. Gustavo Giovannoni, «La restauration des monuments en Italie», La Conservation des monuments d'art et d'histoire, Paris, publication de l'institut de coopération intellectuelle, 1933, p. 60-66.

6. Ibid., «Les moyens modernes de construction appliqués à la restauration des monuments », $p$. 180.

7. Ibid., p. 181.

8. Ibid., « Conclusions de la Conférence », p. 402. I- Doctrines. Principes généraux.

9. Ibid., p. 404.

10. Archives de la Médiathèque de l'architecture et du Patrimoine (AMP), procès-verbaux de la commission des monuments historiques (PV), numéro d'inventaire $\left(\mathrm{N}^{\circ}\right)$ 80/15/24, du 27 juin 1919, « Travaux exécutés dans les édifices des régions libérées ».

11. C'est à dire la restauration qui transforme la matière de l'œuvre d'art en utilisant les mêmes matériaux que ceux des éléments historiques et en recomposant la décoration disparue.

12. Françoise Bercé, Des monuments historiques au Patrimoine, du XVIII siècle à nos jours ou "Les égarements du cœur et de l'esprit », Paris, Flammarion, 2000, p. 62.

13. Giuseppe Cristinelli, La carta di Cracovia 2000. Principi per la conservazione e il restauro del patrimonio costruito, Venezia, Marsilio, 2002.

14. La charge d'« architecte ordinaire" est instituée en 1897. Les financements, liés dans un premier temps à l'approbation de la Commission, sont, à partir de 1912, alloués directement à l'architecte sur le territoire, celui-ci devant être en mesure d'en effectuer l'entretien d'une manière autonome, à travers des visites constantes aux monuments.

15. Maria Piera Sette, «Un nodo del restauro : la reintegrazione fra notorietà, distinguibilità, reversibilità ", Opus Quaderno di storia dell'architettura e restauro , $\mathrm{n}^{\circ}$ 2, 1990, p. 243-248.

16. AMP, $\mathrm{N}^{\circ} 80 / 58 / 01,10$ mai 1920, Paul Léon, leçon inaugurale.

17. Émile Brunet, "Les édifices classés de l’Aisne ", La Construction moderne, 24 mars 1929, p. 298-307.

18. Les Monuments historiques de la France, 1939, p. 49.

19. Jules Formigé, Le Trophée des Alpes (La Turbie). Fouilles et monuments archéologiques en France métropolitaine (supplément à «Gallia» II), Paris, Centre national de recherche scientifique, 1949, p. 76. 
20. Emmanuel Gonze, » L'église Saint-Pierre-de-Roye », l'Art sacré, $n^{\circ}$ 9, mars 1936, p. 76-81, cité par Jean-Charles Cappronnier, «Les églises reconstruites après la Grande Guerre ", Reconstructions en Picardie après 1918, Catalogue de l'exposition aux Archives départementales de l'Aisne, 16 septembre 2000-15 janvier 2001, Paris, Éditions de la Réunion des musées nationaux, 2000, p. 170.

21. AMP, PV, $\mathrm{n}^{\circ}$ 80/15/27, du 26 octobre 1926.

22. François Loyer, Histoire de l'architecture française de la Révolution à nos jours, Paris, Éditions du Patrimoine, 1995, vol. 3, p. 303.

23. AMP, PV, $\mathrm{n}^{\circ} 80 / 15 / 26$, du 14 février 1925, Nasbinals - église.

24. AMP, PV, n ${ }^{\circ}$ 80/15/28, du 27 mars 1931, Paris - Hôtel de Sens.

25. AMP, PV, $\mathrm{n}^{\circ}$ 80/15/29, du 22 mars 1935.

26. AMP, PV, $\mathrm{n}^{\circ}$ 80/15/26, du 29 mai 1925 et 13 novembre 1925, Carcassonne (Aude) Construction d'un hôtel dans la Cité.

27. AMP, PV, n ${ }^{\circ}$ 80/15/29, du 13 janvier 1939, Paris - École nationale de Mines, ancien hôtel Vendôme.

28. AMP, PV, no 80/15/26, du 30 mars 1925, Noyon.

29. Ibid.

30. AMP, $P V, n^{\circ} 80 / 15 / 26$, du 14 mai 1926, Reims - Cathédrale.

31. AMP, PV, $\mathrm{n}^{\circ}$ 80/15/25, du 03 mars 1922, Tours - Cathédrale.

32. AMP, PV, $n^{\circ}$ 80/15/28, du 22 mai 1931.

33. "Catalogue de l'exposition des monuments historiques", Les Monuments historiques de la France, deuxième année, 1937, p. 58.

34. AMP, PV, $\mathrm{n}^{\circ}$ 80/15/29, du 09 décembre 1938, Paris - Église Saint Gervais.

35. AMP, $\mathrm{n}^{\circ} 80$ août 08 , Paul Deschamps, Notes pour l'Exposition internationale, 17 p., Exposition Universelle 1937.

36. AMP, $n^{\circ}$ 81/50/54, du 17 mai 1926, Pierre Paquet, travaux au Mont Saint-Michel.

37. Paul Léon, «La restauration des monuments en France ", La Conservation des monuments d'art et d'histoire, Paris, Publication de l'Institut de Coopération Intellectuelle, 1933, p. 56.

38. AMP, PV, $n^{\circ}$ 80/58/2, du 05/02/1921, Charles Genuys, leçon inaugurale à l'École de Chaillot.

\section{RÉSUMÉS}

Les années vingt et trente du siècle dernier constituent une période fondamentale pour le développement de la théorie de la restauration. L'analyse de la position française, à travers la lecture des procès-verbaux de la Commission des monuments historiques et des décisions qui sont prises par cette dernière, présente un scénario complexe et articulé. D'une part, les interventions sont effectuées en continuité avec une position héritée du XIX ${ }^{\mathrm{e}}$ siècle, guidées par les théories de Viollet-le-Duc et, d'autre part, de nouveaux principes s'affirment, discutés à l'échelon international, où la France, contrairement à ce qui a été souvent soutenu, joue un rôle de premier plan. Il semble que s'esquisse, entre les lignes d'une doctrine jamais clairement explicite, une alternative aux théories de Giovannoni, dans laquelle tradition et innovation trouvent un heureux équilibre. 
The 1920s and 1930s were a period of crucial importance for developments in the theory of restoration. An analysis of France's position, based on the procès-verbaux of the Commission des monuments historiques and the decisions made at that meeting, reveals a complicated and complexly-structured situation. On the one hand, restorations were carried out ensuring continuity with a nineteenth-century model, based on the theory of Viollet-le-Duc. On the other, new, internationally-discussed principles were emerging, in which, contrary to popular belief, France played a leading role. Reading between the lines of a doctrine that was never clearly explained, there appears to be an alternative to the theories of Giovannoni, characterized by a perfect balance between tradition and innovation.

Die zwanziger und dreißiger Jahre des letzten Jahrhunderts erweisen sich als grundlegender Wendepunkt in der Entwicklung der Theorie der Restaurierung. Die Lektüre der Protokolle ( procès-verbaux) der Commission des Monuments historiques und der in diesem Rahmen getroffenen Entscheidungen gibt einen Einblick in die Komplexität des französischen Standpunktes. Einerseits bleibt die französische Praxis der Restaurierung je nach der Tradition des 19. Jahrhunderts durch die Theorien Viollet-le-Ducs inspiriert, andererseits kommt Frankreich, entgegen weitläufiger Meinung, ein erstrangiger Anteil an den internationalen Debatten zu, die neue Prinzipien entwickeln.Wenn es auch nie deutlich erklärt wird, zeichnet sich doch zwischen den Zeilen eine Alternative zu den Theorien Giovannonis ab, in denen Tradition und Innovation optimal abgewogen werden.

\section{AUTEUR}

\section{STÉPHANE GARNERO}

Stéphane Garnero, est né en 1970. Il a obtenu en 1996 son diplôme d'architecte à l'École polytechnique de Turin avec un projet sur la restauration et l'extension de la Villa della Regina, sous la direction de Roberto Gabetti et de Maurizio Momo. En 2002, il est docteur en restauration avec une thèse sur la restauration en France entre les deux guerres, sous la direction de Maurizio Momo et de François Loyer. En 2005, il devient « architecte du patrimoine », après avoir suivi les cours de l'École de Chaillot. Il travaille sur la restauration d'édifices, dont il publie, comme collaborateur de Maurizio Momo et Gabetti \& Isola, les résultats dans Abitare n 375/1998 « Restauration du Dôme de Turin et nouvelle sacristie pour l'ostension du Saint Suaire » et dans Aion $\mathrm{n}^{\circ}$ 5/2004, « Restauration de la Palazzina di Caccia di Stupinigi ». Il participe à la restauration de la Villa della Regina et publie « Un dossier per la Villa. Documentazione dagli archivi storici » dans Villa della Regina, diario di un cantiere in corso, Torino, Umberto Allemandi \& C., 1997, pp. 79-95 ; « Nuovi disegni per la Villa », Ibid., pp. 95-99 ; « Dai danni di guerra agli anni sessanta. Documenti sugli interventi di manutenzione », Ibid., pp. 109-117 et un texte avec Rebecca De Marchi : La Vigna del cardinal Maurizio : il racconto di villa della Regina, Torino, edizione Beppe Grande, 1999. Il est collaborateur des cours de Maurizio Momo, de Giovanni Durbiano et de Elena Tamagno à l'École polytechnique de Turin. Adresse électronique : s.garnero@fastwebnet.it 